https://doi.org/10.15407/ujpe63.3.232

YA.I. KOLESNICHENKO, V.V. LUTSENKO, T.S. RUDENKO

Institute for Nuclear Research, Nat. Acad. Sci. of Ukraine

(47, Prosp. Nauky, Kyiv 03028, Ukraine; e-mail: tima_rudenko@ukr.net)

\title{
AN EQUATION OF THE QUASILINEAR THEORY WITH WIDE RESONANCE REGION
}

\begin{abstract}
An equation of the quasilinear theory is derived. It is based on the same assumptions as the well-known equation in [1]. However, it has another form of the quasilinear operator, which does not contain the longitudinal wavenumber. Due to this, characteristics of the derived equation determine the routes of a quasilinear evolution of the particle distribution function, even when the resonance region determined by the spectrum of longitudinal wavenumbers is wide. It is demonstrated that during the ion acceleration by the ion cyclotron resonant heating, (i) the change of the longitudinal ion energy can be considerable and (ii) the increase of the particle energy may well exceed the increase described by characteristics of the Kennel-Engelmann equation (which are shown, in particular, in [10]), because these characteristics represent the ways of the quasilinear diffusion only when the resonance region is narrow.
\end{abstract}

Keywords: quasilinear theory, resonance region, ion cyclotron resonant heating, resonance, wave frequency, ion acceleration.

The effects of waves in plasmas on the particle distributions in the phase space can be described by the quasilinear (QL) theory. The equations of this theory were derived and analyzed in many works. In the simplest case where the approximation of a straight magnetic field is valid, the $\mathrm{QL}$ equations in various forms were derived many years ago. The most known QL equation was proposed by Kennel and Engelmann (K\&E) [1]. Later, quasilinear equations involving the inhomogeneity of the magnetic field of toroidal fusion devices were derived and analyzed (see, e.g., [28]). Nevertheless, many practical applications of the QL theory are associated with the K\&E equation, because it often describes main features of the quasilinear distortion of the particle distribution function, being at the same time rather simple. In particular, studies of various scenarios of the Ion Cyclotron Resonant Heating (ICRH) are relied to a large extent on

(C) YA.I. KOLESNICHENKO, V.V. LUTSENKO, T.S. RUDENKO, 2018

232 the theory in $[9,10]$, which is actually based on the $\mathrm{K} \& \mathrm{E}$ equation.

The K\&E theory describes the influence of waves on the particles, which satisfy the resonance condition

$\omega_{k}-l \omega_{B}-k_{\|} v_{\|}=0$

where $\omega_{k}$ is the wave frequency, $k_{\|}$is the longitudinal wavenumber, $v_{\|}$is the particle longitudinal velocity, $\omega_{B}$ is the particle gyrofrequency, and $l$ is an integer. Depending on the waves destabilized or launched by an antenna, Eq. (1) determines different resonance regions in the $v_{\|}$space. For instance, when the waves have wavenumbers in the range $k_{\| \min }<k_{\|}<k_{\| \max }$, whereas their frequency is monochromatic $\left(\omega_{k}=\omega\right)$ and $\omega_{B}$ is given, the width of the resonance region $\left(\Delta v_{\|} \equiv v_{\| \max }-v_{\| \min }\right)$ is determined by the condition $k_{\| \min } v_{\| \max }=k_{\| \max } v_{\| \text {min }}$, from which it follows that $\Delta v_{\|} / v_{\| \min }=\Delta k_{\|} / k_{\| \min }$. Thus, the resonance region is wide, when $\Delta k_{\|} / k_{\| \text {min }}$ is not small, which is typically the case during ICRH.

ISSN 2071-0194. Ukr. J. Phys. 2018. Vol. 63, No. 3 
Experiments with ICRH confirm that the resonance region is wide: first, ICRH affects thermal particles with different pitch angles, which makes the heating efficient. Second, ICRH often strongly accelerates a group of ions, by leading to the formation of a high-energy tail on the distribution function of heated ions. For instance, a non-Maxwellian tail consisting of $\alpha$-particles with the energies up to several $\mathrm{MeV}$ was observed in the JET tokamak [11]. Although ICRH increases mainly the particle energy across the magnetic field, $\mathcal{E}_{\perp}$, the longitudinal energy, $\mathcal{E}_{\|}$, changes considerably, too, when $\Delta \mathcal{E}_{\perp} \gg T$, with $T$ being the plasma temperature.

Thus, it is of importance to know the route in the phase space of the QL process, when the resonance region is wide. However, characteristics of the QL equation in Refs. $[1,9,10]$ (which determines the QL route) are not applicable in this case. They are given by

$$
\left(v_{\|}-\frac{\omega_{k}}{k_{\|}}\right)^{2}+v_{\perp}^{2}=\text { const }
$$

where $v_{\perp}$ is the particle velocity across the magnetic field. Equation (2) describes semicircles in the $\left(v_{\|}, v_{\perp}\right)$ plane, which are shown in Fig. 4 in [9] and Fig. 17-2 in [10]. This equation was obtained by integrating a differential equation in the assumption of $\omega_{k} / k_{\|}=$const. Therefore, it was emphasized in $[1,9,10]$ that only small parts of the circles around $v_{\|}=\omega_{k} / k_{\|}$represent the direction of the quasilinear diffusion. The rest, i.e., the main part of the characteristics shown is meaningless.

In this work, a QL equation in a form convenient for the study of QL processes with the arbitrary width of the resonance region determined by the $k_{\|}$spectrum is derived. Its characteristics describing the route of acceleration of a group of ions during ICRH is considered and compared with K\&E characteristics.

\section{Quasilinear Equation}

Let us write a particle distribution function in the form $F=f_{0}+\tilde{f}$, where $f_{0}$ and $\tilde{f}$ are the equilibrium and disturbed parts of the distribution, respectively. The equilibrium distribution function depends on the integrals of motion, which are the particle energy $(\mathcal{E})$ and the magnetic moment $\left(\mu_{p}\right)$. But when the effects of inhomogeneity of the magnetic configuration are negligible, we can take $f_{0} \equiv f_{0}\left(\mathcal{E}, \mathcal{E}_{\perp}\right)$. The perturbation $\tilde{f}$ is determined by the linearized colli- sionless kinetic equation. It can be written as follows:

$\tilde{f}(\boldsymbol{r}, \boldsymbol{v}, t)=\sum_{\boldsymbol{k}} f_{\boldsymbol{k}} e^{-i \omega_{k} t+i \boldsymbol{k} \boldsymbol{r}}=$

$=-\frac{e}{M} \int_{-\infty}^{t}\left(\tilde{\boldsymbol{E}}+\frac{1}{c} \boldsymbol{v} \times \tilde{\boldsymbol{B}}\right) \nabla_{\boldsymbol{v}} f_{0} d \tau$,

where the integral is taken along particle orbits, $\tilde{\boldsymbol{E}}$ and $\tilde{\boldsymbol{B}}$ are the perturbed parts of the electric field and magnetic field, respectively, $v_{x}=$ $=v_{\perp} \cos \alpha, v_{y}=v_{\perp} \sin \alpha, \dot{\alpha}=-\omega_{B}, k_{x}=k_{\perp} \cos \psi$, $k_{y}=k_{\perp} \sin \psi, e$ is the electric charge, and $\mathrm{M}$ is the particle mass. Then $\boldsymbol{v} \cdot \boldsymbol{E}_{\boldsymbol{k}}=v_{\perp}\left(E_{+} e^{-i \alpha}+E_{-} e^{i \alpha}\right)+$ $+v_{\|} E_{\|}$, with $E_{ \pm}=E_{x} \pm i E_{y}$. Using this relation and

$$
\begin{aligned}
& e^{-i \xi \sin (\alpha-\psi)}=\sum_{l} J_{l}(\xi) e^{-i l(\alpha-\psi)}, \\
& e^{ \pm i \alpha} e^{-i \xi \sin (\alpha-\psi)}=\sum_{l} J_{l \pm 1}(\xi) e^{-i l(\alpha-\psi)} e^{ \pm i \psi},
\end{aligned}
$$

where $J_{l}(\xi)$ is the Bessel function, $\xi=k_{\perp} \rho_{\perp}, \rho_{\perp}=$ $=v_{\perp} / \omega_{B}$ is the Larmor radius, we obtain

$f_{\boldsymbol{k}}=-e \sum_{l} e^{i \xi \sin (\alpha-\psi)-i l(\alpha-\psi)} \times$

$\times \int_{0}^{\infty} d t_{1}\left(\boldsymbol{G} \cdot \boldsymbol{E}_{\boldsymbol{k}} \hat{\Pi} f_{0}+\boldsymbol{G}_{\perp} \cdot \boldsymbol{E}_{\perp \boldsymbol{k}} \frac{1}{i \omega_{\boldsymbol{k}}} \frac{\partial f_{0}}{\partial \mathcal{E}_{\perp}} \frac{d}{d t}\right) e^{i \Omega_{l} t_{1}}$

where $\Omega_{l}=\omega_{\boldsymbol{k}}-l \omega_{B}-k_{\|} v_{\|}$,

$\boldsymbol{G} \cdot \boldsymbol{E}_{\boldsymbol{k}}=\boldsymbol{G}_{\perp} \cdot \boldsymbol{E}_{\perp \boldsymbol{k}}+v_{\|} E_{\|} J_{l}$,

$\boldsymbol{G}_{\perp} \cdot \boldsymbol{E}_{\perp \boldsymbol{k}}=v_{\perp}\left(E_{+} J_{l-1} e^{-i \psi}+E_{-} J_{l+1} e^{i \psi}\right)$,

$\hat{\Pi}=\frac{\partial}{\partial \mathcal{E}}+\frac{l \omega_{B}}{\omega_{\boldsymbol{k}}} \frac{\partial}{\partial \mathcal{E}_{\perp}}$.

Note that $\boldsymbol{G}_{\perp} \cdot \boldsymbol{E}_{\perp \boldsymbol{k}}$ can be written in the form

$\boldsymbol{G} . \perp \boldsymbol{E}_{\perp \boldsymbol{k}}=\frac{l J_{l}}{\xi} v_{\perp} E_{1}+i \frac{\partial J_{l}}{\partial \xi} v_{\perp} E_{2}$,

where $E_{1}$ and $E_{2}$ are vector components, $E_{1}=\boldsymbol{E}_{\boldsymbol{k}} \cdot \boldsymbol{e}_{1}$, $E_{2}=\boldsymbol{E}_{\boldsymbol{k}} \cdot \boldsymbol{e}_{2}$, the unit vectors are defined by $\boldsymbol{e}_{1}=$ $=\boldsymbol{k}_{\perp} / k, \boldsymbol{e}_{2}=\boldsymbol{e}_{3} \times \boldsymbol{e}_{1}, \boldsymbol{e}_{3}=\boldsymbol{B}_{0} / B_{0}$.

Assuming $\Omega_{l} \rightarrow \Omega_{l}+i 0$ (which corresponds to the Landau rule), we calculate the integrals in (5):

$$
\int_{0}^{\infty} d t_{1} e^{i \Omega_{l} t_{1}}=i \frac{P}{\Omega_{l}}+\pi \delta\left(\Omega_{l}\right), \int_{0}^{\infty} d t_{1} \frac{d}{d t_{1}} e^{i \Omega_{l} t_{1}}=-1 .
$$




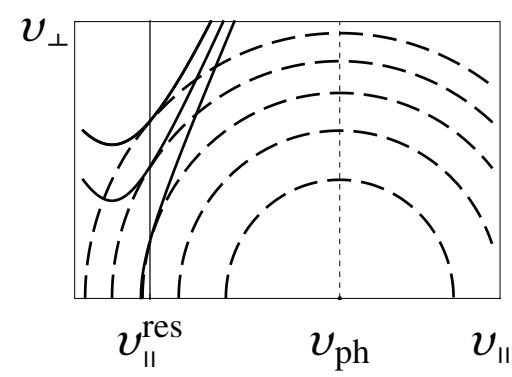

Fig. 1. Characteristics determined by the K\&E equation (2) (dashed curves) and Eq. (20) (thick solid lines) in the $\left(v_{\|}, v_{\perp}\right)$ space. In calculations, we took $\tilde{\omega}=1.2$ and $l=1$, so that $v_{\|}^{\mathrm{res}} / v_{\mathrm{ph}}=1 / 6$ according to $(23)$

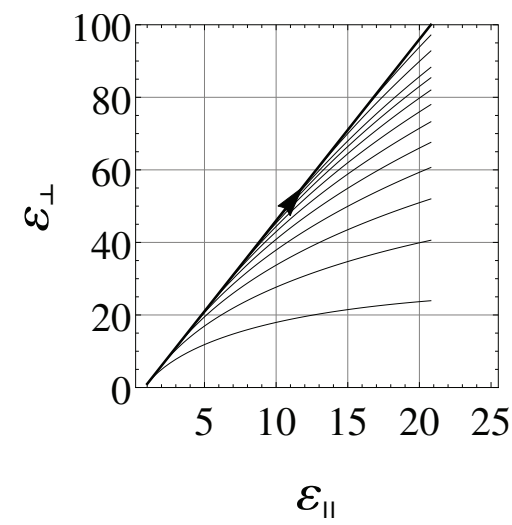

Fig. 2. The route of the QL acceleration of resonant ions during ICRH (bold straight line) and the K\&E characteristics (2) (thin lines) in the $\left(\mathcal{E}_{\|}, \mathcal{E}_{\perp}\right)$ space. In calculations, we took $\tilde{\omega}=1.2$ and $l=1$, as in Fig. 1, but different wave phase velocities were used to calculate the different $\mathrm{K} \& \mathrm{E}$ characteristics. Only those parts of the K\&E characteristics that lie above the points, where they touch the bold straight line, are shown. The acceleration route is determined by Eq. (20), the arrow shows the direction of the QL acceleration of particles with the initial energies $\mathcal{E}_{\|}=1$ and $\mathcal{E}_{\perp}=1$ (the energy is convenient to be normalized to the plasma temperature). It is assumed that the spectrum of $k_{\|}$provides the wave-particle interaction for the acceleration up to the energy $\mathcal{E}_{\perp}=100$

It follows from Eqs. (5) and (10) that the distribution function consists of the resonant and non-resonant parts: $f=f_{r}+f_{n}$. The resonant part is

$f_{\boldsymbol{k}, r}=-\pi e \sum_{l} e^{i \xi \sin (\alpha-\psi)-i l(\alpha-\psi)} \boldsymbol{G} \cdot \boldsymbol{E}_{\boldsymbol{k}} \hat{\Pi} f_{0} \delta\left(\Omega_{l}\right)$.

Only this part of $f_{\boldsymbol{k}}$ contributes to the equation, which determines the evolution of the equilibrium dis- tribution function caused by the waves:

$$
\frac{\partial f_{0}}{\partial t}=-\frac{e}{M} \sum_{\boldsymbol{k}} \oint \frac{d t}{\tau_{B}} \nabla_{\boldsymbol{v}}\left(\boldsymbol{E}_{\boldsymbol{k}}+\frac{1}{c} \boldsymbol{v} \times \boldsymbol{B}_{\boldsymbol{k}}\right) f_{\boldsymbol{k}, r}^{*},
$$

where $\tau_{B}$ is the gyroperiod, $d t / \tau_{B}=-d \alpha /(2 \pi)$,

$\nabla_{\boldsymbol{v}}=M \boldsymbol{v} \frac{\partial}{\partial \mathcal{E}}+M \boldsymbol{v}_{\perp} \frac{\partial}{\partial \mathcal{E}_{\perp}}+\frac{\boldsymbol{B} \times \boldsymbol{v}}{B v_{\perp}^{2}} \frac{\partial}{\partial \alpha}$

Noting that $d^{3} v=M^{-2} d \mathcal{E} d \mathcal{E}_{\perp} d \alpha /\left|v_{\|}\right|$, we present $\nabla_{\boldsymbol{v}} \cdot \boldsymbol{A}$, with $\boldsymbol{A}=\boldsymbol{A}\left(\mathcal{E}, \mathcal{E}_{\perp}, \alpha\right)$ being an arbitrary vector, in the following form:

$$
\begin{aligned}
& \nabla_{\boldsymbol{v}} \cdot \boldsymbol{A}=M v_{\|}\left(\frac{\partial}{\partial \mathcal{E}} \frac{\boldsymbol{A} \cdot \boldsymbol{v}}{v_{\|}}+\frac{\partial}{\partial \mathcal{E}_{\perp}} \frac{\boldsymbol{A} \cdot \boldsymbol{v}_{\perp}}{v_{\|}}\right)+ \\
& +\frac{\partial}{\partial \alpha} \frac{\boldsymbol{A} \cdot\left(\boldsymbol{B}_{0} \times \boldsymbol{v}\right)}{B_{0} v_{\perp}^{2}}
\end{aligned}
$$

Then, due to the relations

$$
\begin{aligned}
& \int_{0}^{2 \pi} \frac{d \alpha}{2 \pi} e^{i \xi \sin (\alpha-\psi)-i l(\alpha-\psi)}=J_{l}(\xi), \\
& \int_{0}^{2 \pi} \frac{d \alpha}{2 \pi} e^{i \alpha} e^{i \xi \sin (\alpha-\psi)-i l(\alpha-\psi)}=J_{l \pm 1}(\xi) e^{i \psi},
\end{aligned}
$$

equation (12) can be written as

$\frac{\partial f_{0}}{\partial t}=v_{\|} \sum_{\boldsymbol{k}, l} \hat{\Pi} v_{\|}^{-1} D_{\boldsymbol{k}, l} \hat{\Pi} f_{0}$,

where $\hat{\Pi}$ is defined by (8) and

$D_{\boldsymbol{k}, l}=\pi e^{2}\left|\boldsymbol{G} \cdot \boldsymbol{E}_{\boldsymbol{k}}\right|^{2} \delta\left(\Omega_{l}\right)$.

The characteristics of this equation are determined by

$\hat{\Pi} f_{0}=0$.

For the fixed wave frequency, they are given by

$l \omega_{B} \mathcal{E}-\omega_{\boldsymbol{k}} \mathcal{E}_{\perp}=\mathrm{const}$

which transforms to the following relation in the variables $\left(\mathcal{E}_{\|}, \mathcal{E}_{\perp}\right)$ :

$\mathcal{E}_{\|}-\left(\frac{\omega_{\boldsymbol{k}}}{l \omega_{B}}-1\right) \mathcal{E}_{\perp}=$ const.

ISSN 2071-0194. Ukr. J. Phys. 2018. Vol. 63, No. 3 
A similar picture takes place in the framework of the quasilinear theory dealing with times exceeding particle transit/bounce times, as shown in [3].

In contrast to this, according to Refs. $[1,9,10]$, the quasilinear evolution takes place along the curves determined by (2).

Equation (2) represents characteristics of the equation

$k_{\|} \frac{\partial f_{0}}{\partial v_{\|}}+\left(\omega_{\boldsymbol{k}}-k_{\|} v_{\|}\right) \frac{1}{v_{\perp}} \frac{\partial f_{0}}{\partial v_{\perp}}=0$,

which can be written in the variables $\left(\mathcal{E}, \mathcal{E}_{\perp}\right)$ in the form

$\hat{\Pi} f_{0}+\frac{\Omega_{l}}{\omega_{\boldsymbol{k}}} \frac{\partial f_{0}}{\partial \mathcal{E}}=0$.

The second term in this equation is actually the second term of the integrand of Eq. (5), which is associated with the non-resonant part of the distribution function, $f_{\boldsymbol{k}, n}$. The inclusion of the non-resonant term for the description of the QL evolution of resonant particles leads to characteristics (2), which have physical sense only in a vicinity of the particular resonance velocity.

Although Eq. (22) includes non-resonant term, it coincides with Eq. (18) at the points of exact resonance $\left(\Omega_{l}=0\right)$. Therefore, the slope of characteristics at these points is the same in both cases. However, Eqs. (19), (20), and (2) determine different curves. For instance, when $\omega=l \omega_{B}$, Eq. (19) predicts $v_{\|}=$const, which together with the resonance $\Omega_{l}=0$ means that only $v_{\perp}$ changes during the quasilinear evolution of particles with $v_{\|}=0$. On the other hand, it follows from (2) that $v_{\perp}=$ const for the particles with $v_{\|}=0$.

Owing to the coincidence of the slopes of characteristics at the resonance, both (20) and (2) predict approximately the same character of the quasilinear evolution, when the wave spectrum is sufficiently narrow. In order to demonstrate this, let us calculate both families of characteristics for a given wave phase velocity $\left(v_{\mathrm{ph}}=\omega_{k} / k_{\|}\right)$in the $\left(v_{\|}, v_{\perp}\right)$ plane. Because Eq. (20) for the characteristics of our QL equation does not contain $v_{\mathrm{ph}}$, we need the following relation obtained from the resonance condition (1):

$v_{\mathrm{ph}}=v_{\|}^{\mathrm{res}} \frac{\tilde{\omega}}{\tilde{\omega}-l}$, where $\tilde{\omega}=\omega / \omega_{B}, v_{\|}^{\text {res }}$ is the resonance longitudinal velocity. Then we obtain two families of characteristics shown in Fig. 1. We observe a good agreement between these families in a vicinity of $v_{\|}^{\text {res }}$, although the behaviors of the shown curves away from this velocity are very different.

It is of interest to find the QL route of those thermal ions that are strongly accelerated by ICRH. Let us assume that the transversal energy of the particles increases by a factor of 100 (e.g., $\mathcal{E}_{\perp}$ grows from $10 \mathrm{keV}$ to $1 \mathrm{MeV}$ ). Because characteristics (20) are straight lines in the plane $\left(\mathcal{E}_{\|}, \mathcal{E}_{\perp}\right)$, it is convenient to use the space of $\left(\mathcal{E}_{\|}, \mathcal{E}_{\perp}\right)$. For the comparison, we will also calculate characteristics (2). With this purpose, we write (2) as

$\mathcal{E}_{\perp}=\mathcal{E}_{\|}\left(2 \frac{v_{\mathrm{ph}}}{v_{\|}}-1\right)+$ const

where we will eliminate the wave phase velocity by means of the resonance relation (23). The results of calculations are shown in Fig. 2. It follows from this figure that $\mathcal{E}_{\|}$considerably, by a factor of 20 , increases during ICRH. The increase of $\mathcal{E}_{\perp}$ described by the K\&E characteristic going through the starting point $\left(\mathcal{E}_{\|}=\mathcal{E}_{\perp}=1\right)$ is much less than the prescribed magnitude (one hundred). In addition, we conclude that the envelop of the infinite number of $K \& E$ characteristics coincide with the straight line representing the direction of the QL evolution given by (20).

\section{Summary and Conclusions}

A QL equation with characteristics, which do not depend on $k_{\|}$, is derived. They determine the way in the phase space independently of the $k_{\| \text {-spectrum }}$ provided that the following conditions are satisfied. First, the wave frequency should be fixed during the quasilinear evolution of the distribution function, which is the case during RF heating and under some plasma instabilities. Second, the single $l$-term in the QL equation should be mainly responsible for the $\mathrm{QL}$ process. This implies that the wave frequency should be close to a cyclotron harmonic $l \omega_{B}$ with $l \sim 1$ or $\omega$ should be much less than $\omega_{B}$ (in the latter case, the $l=0$ term dominates); otherwise, $\omega$ well exceeds the distance between the cyclotron harmonics, and, hence, the terms with various $l$ equally contribute. Third, the resonance condition should be satisfied. Fourth, the magnitude of $k_{\perp} \rho_{\perp}$ should not 
reach the magnitudes, for which the Bessel functions in the diffusion coefficient vanish. Fifth, the time of the QL diffusion should be less than the times of Coulomb collisions.

It is demonstrated that, during the ion acceleration by ICRH, (i) the change of the longitudinal ion energy can be considerable and (ii) the increase of the particle energy may well exceed that described by the characteristics of the K\&E equation, because these characteristics represent the ways of quasilinear diffusion only when the resonance region is narrow.

It is found that the $k_{\|}$-dependence in the QL characteristics of Refs. $[1,9,10]$ comes from the nonresonant part of the distribution function $f_{k}$ [see Eq. (5)] - the term, which does contribute to the QL process. Therefore, characteristics (19) and (20) appear in a natural way. On the other hand, the $k_{\|}$spectrum plays an important role, being responsible for the fulfillment of the resonance condition: characteristics (19) and (20) represent the route of the QL process provided that the resonance condition is satisfied. Note that, in reality, the $k_{\|}$spectrum is discrete $\left[k_{\|}=(m \iota-n) / R\right.$ is determined by the mode numbers $m$ and $n$ ]. This implies that the finite wave amplitudes leading to broadening the $\delta\left(\Omega_{l}\right)$-function play an important role, by providing the overlap of resonances with different longitudinal wavenumbers.

Only the resonant particles are affected by waves in the QL theory [although non-resonant wave-particle interaction can lead to some effects, see, e.g., [12]. Therefore, because the the slope of K\&E characteristics at the resonance points coincides with that given by (19) and (20), the K\&E equation and the equation derived in this work are equivalent. It is a matter of convenience, which QL equation should be used. As seen from Fig. 2, the envelope of the infinite number of $\mathrm{K} \& \mathrm{E}$ characteristics calculated for different wave phase velocities coincides with the straight line determined by $(20)$.

Note that, as we already mentioned at the beginning of this work, the limited value of the characteristics in the form of circles was recognized in [1]. Therefore, the authors of [1] "constructed" a new characteristic equation instead of their equation (4.7) [our equation (21)] and finally arrived at Eq. (4.11) [our equation (20)]. In order to obtain (4.11), they changed the coefficients in the differential equation (4.7), by using the resonance condition, which is not correct. Characteristic curves and resonance curves are independent curves. Therefore, the procedure of construction of the characteristic equation used in [1] is not justified. For this reason, it was not clear $a$ priori, whether the characteristics given by Eq. (4.11) are correct. This may explain why only the circles [rather than curves described by (4.11)] were shown in [1] and in subsequent T. Stix's publications. On the other hand, we actually have proved that Eq. (4.11) is correct, because this equation [our Eq. (20)] represents a solution of (18), which directly follows from the QL equation derived in this work.

It is known that the ion acceleration during ICRH reduces the particle pitch $\chi \equiv v_{\|} / v$ and eventually can lead to the transformation of passing particles to trapped ones. However, characteristics of our equation in the space $(\lambda, \mathcal{E})$, with $\lambda=\mathcal{E}_{\perp} / \mathcal{E}=1-\chi^{2}$, are given by

$\mathcal{E}\left(\lambda-l \tilde{\omega}^{-1}\right)=$ const.

This equation predicts the existence of the separatrix $\lambda=l \tilde{\omega}^{-1}$ between the regions with $d \lambda / d \mathcal{E}>0$ (where the ICRH decreases $\chi$ ) and the region with $d \lambda / d \mathcal{E}<0$ (where the ICRH increases $\chi$ ). This opens a possibility to improve the confinement of fast ions (which is a problem for stellarators) by transforming trapped fast particles (which may be not confined) to passing ones by means of the ICRH [13].

However, a question may arise whether the approximation of homogeneous magnetic field adopted in our theory is applicable to plasmas in tokamaks and stellarators. In this connection, we note that Eq. (25) coincides with a corresponding equation of the works employing the bounce/transit time averaging. This is what one could to expect. The same equation can be obtained, by using the symmetric properties of the resonance term in the particle Lagrangian (i.e., not using the quasilinear theory), like this was done in, e.g., [14]. In addition, Eq. (25) follows from the orbitaveraged QL equation in the general form proposed by Kaufman [2], as shown in [6]. Of course, the inhomogeneity of the magnetic field plays, in reality, an important role by leading to specific particle orbits, restricting the region of the resonant wave-particle interaction through resonance (1), which becomes local, and by leading to the appearance of global (orbitaveraged) resonances. All these factors, however, do not affect the route of the quasilinear diffusion, but influence the magnitude of the diffusion coefficient. Nevertheless, in some cases, the coefficient of diffusion

ISSN 2071-0194. Ukr. J. Phys. 2018. Vol. 63, No. 3 
in a homogeneous magnetic field coincides with that in a tokamak field. For instance, as shown in [4], the diffusion coefficient for passing the particles in tokamaks in a certain case reduces to the Stix result [10] obtained by the simple flux-surface averaging of Eq. (17).

In summary, a QL theory equation equivalent to the $K \& E$ equation is derived by means of a streamlined method. This equation has an advantage, by directly giving QL routes valid even for wide resonance regions instead of the well-known circles in the $\left(v_{\|}, v_{\perp}\right)$ plane [shown, in particular, in books by T.H. Stix [10] (Fig. 17-2) and by A.I. Akhiezer et al. [15] (Fig. 9.2.1)], which have sense only in a vicinity of the particular wave phase velocity.

The work was supported by the Project No. PL15/17 of NASU.

1. C.F. Kennel, F. Engelmann. Velocity space diffusion from weak plasma turbulence in a magnetic field. Phys. Fluids 9, 2377 (1966).

2. A.N. Kaufman. Quasilinear diffusion of an axisymmetric toroidal plasma. Phys. Fluids 15, 1063 (1972).

3. V.S. Belikov, Ya.I. Kolesnichenko. Derivation of the quasilinear theory equations for the axisymmetric toroidal systems. Plasma Phys. 24, 61 (1982).

4. V.S. Belikov, Ya.I. Kolesnichenko. Quasilinear theory for a tokamak plasma in the presence of cyclotron resonance. Plasma Phys. Control. Fusion 36, 1703 (1994).

5. L.-G. Eriksson, P. Helander. Monte Carlo operators for orbit-averaged Fokker-Planck equations. Phys. Plasmas 1, 308 (1994).

6. L.-G. Eriksson, M.J. Mantsinen, T. Hellsten, J. Carlsson. On the orbit-averaged Monte Carlo operator describing ion cyclotron resonance frequency wave-particle interaction in a tokamak. Phys. Plasmas 6, 513 (1999).

7. P.J. Catto, J. Lee, A.K. Ram. A quasilinear operator retaining magnetic drift effects in tokamak geometry. J. Plasma Phys. 83, 905830611 (2017).
8. A. Bècoulet, D.J. Gambier, A. Samain. Hamiltonian theory of the ion cyclotron minority heating dynamics in tokamak plasmas. Phys. Fluids B 3, No. 1, 137 (1991).

9. T.H. Stix. Fast-wave heating of a two-component plasma. Nucl. Fusion 15, 737 (1975).

10. T.H. Stix. Waves in Plasmas (Springer, 1992).

11. M.J. Mantsinen et al. Alpha-tail production with ioncyclotron-resonance heating of ${ }^{4} \mathrm{He}$-beam ions in JET plasmas. Phys. Rev. Lett. 88, 105002 (2002).

12. A.A Galeev, R.Z. Sagdeev. Nonlinear plasma theory. In: Reviews of Plasma Physics, Vol. 7, edited by M.A. Leontovich (Consultants Bureau, 1979).

13. Ya.I. Kolesnichenko, V.V Lutsenko, T.S. Rudenko, H. Helander. Ways to improve the confinement of fast ions in stellarators by RF waves: General analysis and application to Wendelstein 7-X. Nucl. Fusion 57, 66004 (2017).

14. Ya.I. Kolesnichenko, R.B. White, Yu.V. Yakovenko. Mechanisms of stochastic diffusion of energetic ions in spherical tori. Phys. Plasmas 9, 2639 (2002).

15. A.I. Akhiezer, I.A. Akhiezer, R.V. Polovin, A.G. Sitenko, K.N. Stepanov. Plasma Electrodynamics, Vol. 2 (Pergamon Press, 1975).

Received 29.09.17

Я.І. Колесниченко, В.В. Луценко, Т.С. Руденко

РІВНЯННЯ КВАЗІЛІНІЙНОЇ ТЕОРІї

3 ШИРОКОЮ РЕЗОНАНСНОЮ ОБЛАСТЮ

$\mathrm{P}$ е $з$ ю м е

Отримано рівняння квазілінійної теорії, яке базується на тих самих припущеннях, що i добре відоме рівняння Кеннеля-Енгельмана. Проте форма квазілінійного оператора у отриманому рівнянні не містить поздовжнього хвильового числа. Завдяки цьому характеристики отриманого рівняння визначають шлях квазілінійної еволюції функції розподілу частинок навіть у випадку широкої резонансної області, яка залежить від спектра поздовжніх хвильових чисел. Продемонстровано, що під час прискорення iонів при іонно-циклотронному резонансному нагріванні, поперше, може значно змінюватися поздовжня енергія іонів i, по-друге, збільшення енергії частинок може перевищувати значення, яке визначається характеристиками рівняння Кеннеля-Енгельмана, оскільки останні описують шлях квазілінійної дифузії лише у випадку вузької резонансної області. 